\title{
MISCELLANEA GEOGRAPHICA
}

WARSZAWA 1986

\author{
Mirosława Czerny
}

\section{ACTIVIDADES ECONÓMICAS EN LOS CENTROS POBLACIONALES}

Uno de los aspectos de los estudios sobre asentamientos humanos rurales y urbanos en la región de Las Huastecas ha sido investigar el papel que estos centros de población juegan en la región. Como base de este tipo de estudios nos ha servido tanto la información sobre la existencia en dichos centros de servicios (básicos y especializados), industria, artesanía, asi como la cantidad de personas empleadas en estos sectores de la economía. Material que nos servirá para sacar conclusiones sobre las posibilidades del empleo en la región estudiada y para delimitar las zonas en donde la emigración temporal o estable está más fuertemente marcada.

Los estudios están basados en datos obtenidos por medio de una encuesta "Inventario sobre aspectos socio-económicos de la localidad". Tal encuesta ha sido aplicada en 33 puntos poblacionales (que incluyen localidades rurales, semiurbanas y urbanas).

La información sobre el número total de los empleados y sobre el número de las entidades del empleo ha sido agrupada de la manera siguiente (gráfica 1):

1. Servicios comerciales (repartidos entre 12 tipos de establecimientos comerciales),

2. Otros servicios básicos (12 tipos)

3. Servicios especializados (11 tipos)

4. Empresas productivas (divididos entre empresas industriales y artesanales)

5. Infraestructura técnica (de tres distintos tipos)

Si bien es cierto que los resultados obtenidos de las entrevistas con autoridades locales y con los habitantes de los pueblos visitados por nosotros no han sido del todo completos, si son desde el punto de vista del objeto principal del trabajo, lo suficientemente detallados para los estudios de tipo comparativo mencionados anteriormente.

Aunque no ha sido posible conseguir datos estadisticos sobre el número del empleo en las activiidades de construcción y sobre el número de empresas de construcción, en el presente análisis se menciona el papel que este sector juega en la economía de algunas ciudades. 
Los puntos poblacionales donde se han aplicaido encuestas difieren entre si, a veces muy significamente, desde el punto de vista del número de habitantes. Las diferencias oscilan entre 300 habitantes en algunas zonas rurales hasta 50000 habitantes que viven en la ciudad más grande, de las estudiadas - en Cerro Azul (Cuadro 1.).

Según el análisis, la distribución de las actividades económicas muestra grandes disproporciones entre los respectivos territorios (y/o subregiones).

Se ha aplicado una simple clasificación de los asentamientos humanos tomando como criterio principal el número de servicios así como las actividades productivas existentes en cada lugar estudiado. Se ha tomado en consideración la información sobre dos componentes: 43 categorías de empresas productivas y no productivas y 33 puntos polblacionales estudiados. La ordenación o mejor dicho la permutación primero de los renglones y después de las columnas ha permitido obtener una visión simplificada del estado inicial. El resultado ha sido la obtención de cuatro grupos de elementos:

1. El grupo I - constituido por centros poblacionales que cuentan con una o dos categorías.

2. El grupo II - o sea los centros poblacionales donde el número de categorias oscila entre tres y diez.

3. El grupo III - aquellos centros poblacionales donde el número de categorías se establece entre 11 y 15.

4. El grupo IV - donde en los centros poblacionales se encuentran más de 15 categorías.

Ad. 1. Dentro del primer grupo, peor dotado de cualquier tipo de la actividad no agrícola se encuentran Palma Morelos (no tenemos datos sobre el número de habitantes), Zapotitlán (400 habitantes) y La Lima (600 habitantes). En cadia localidad de este grupo se encuentra una escuela primaria. En los dos primeros hemos encontrado pequeñas tiendas donde se venden productos alimenticios, refrescos y otros artículos necesarios para el hogar. En ambos casos el grupo familiar se encuentra al frente de estas tienidas. En Palma Morelos hay también un carpintero.

Ad. 2. Dentro del segundo grupo se encuentran: El Chote, Calera, Tierra Blanca, Zaragoza, Chinampa de Gorostiza, Raya Obscura, Carmona y Valle, Colomán y El Palmar Prieto. El número de poiblación en estas localidlades es muy desigual encontrándose tanto localidades de 300 como 5500 habitantes (éste último lo constituye el caso de Chinampa de Gorostiza). Tanto las localidades enumeradas en el punto 1 como las de? punto 2 se clasifican como rurales (a veces son pequeños ejidos) donde toda la actividad no agrícola está limitada practicamente sólo al pequeño comercio. 


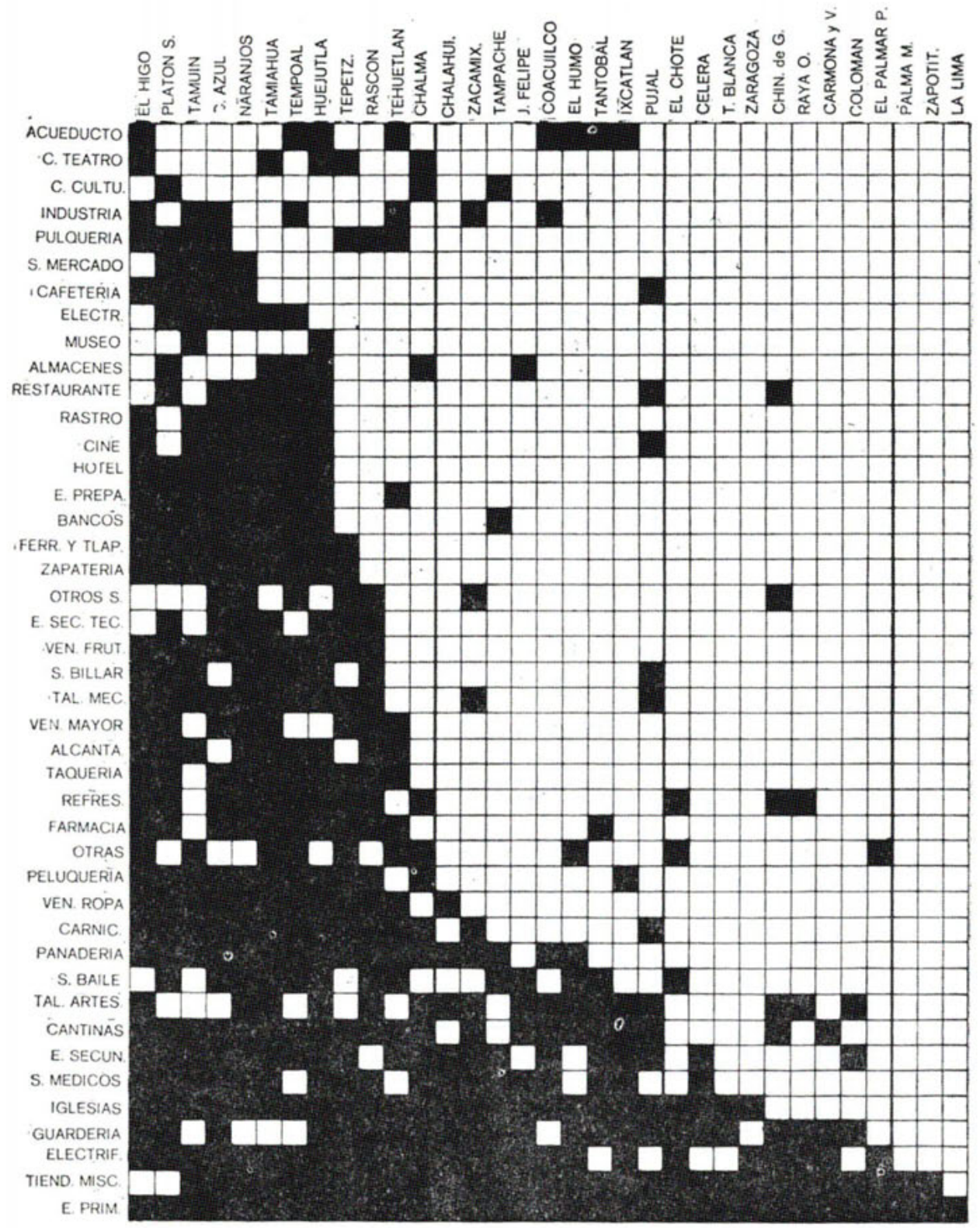

Gráficas I y II

Localidades: 1. Tamiahua, 2. Tampache, 3. Raya Obscura, 4. Palma Morelos, 5. Cerro Azul, 6. Juan Felipe, 7. Colomán, 8. Chinampa đe Gorostiza, 9. Carmona y Valle, 10. Naranjos, 11. Zaragoza, 12. Zacamixtle, 13. Zapotitlán, 14. El Humo, 15. Tierra Blanca, 16. Tepetzintla, 17. Coacuilco, 18. Ixcatlán, 19. Huejutla, 20. Chalahuiyapa, 21. Tehuetlãn, 22. Chalma, 23. Platón Sánchez, 24. Tempoal, 25. EI Higo, 26. El Chote, 27. El Palmar Prieto, 28. Tamuín, 29. Calera, 30. La Lima, 31. Pujal, 32. Tantóbal, 33. Rascón. 


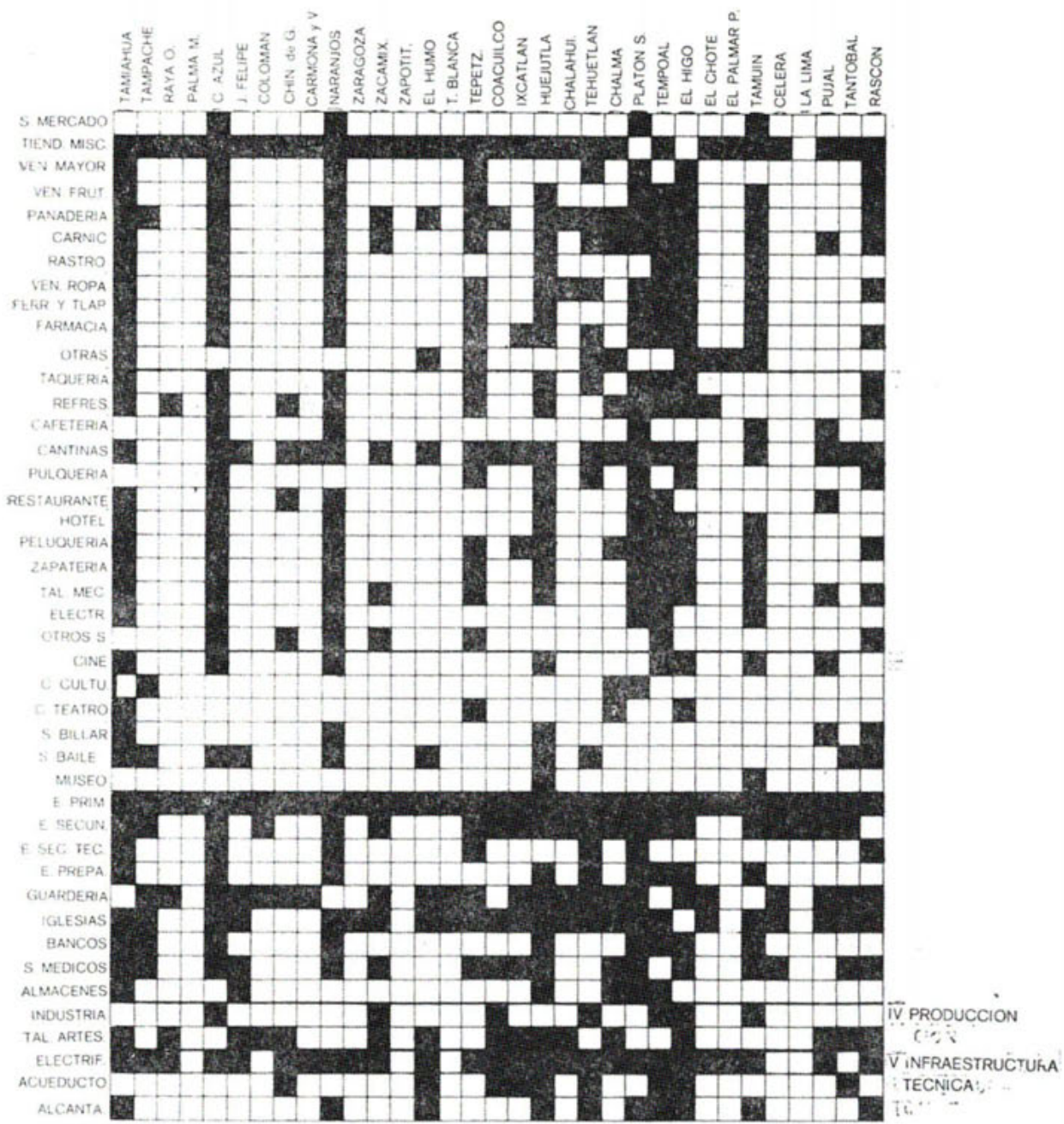

Categorías de las actividades productivas y servicios:

\section{Comercio}

1. Supermercado, 2. Tiendas misceláneas, 3. Venta al por mayor, 4. Venta de frutas y legumbres, 5. Panadería, 6. Carnicería, 7. Rastro, 8. Venta de ropa, 9. Ferretarias y tlapalerías, 10. Farmacia (botica), 11. Otras tiendas

II. Otros servicios básicos

12. Taqueria, 13. Refresquería, 14. Cafetería, 15. Cantinas, 16. Pulquerias y/o Fondas y/o Loncherias, 17. Restaurante, 18. Hotel, 19. Pulquería, 20. Zapatería, 21. Talleres mecántcos y automotrices, 22. Electricistas, 23. Otros servicios de este tipo.

III. Servicios especializados (culturales, educación, salud)

24. Cine (teatro), 25. Campaña de servicios culturales, 26. Club de teatro, 27. Sala de blllar, 28. Sala de baile, 29. Museo, 30. Escuela primaria, 31. Escuela secundaria, 32. Escuela secundaria técnica, 33. Escuela preparataria, 34. Guarderías, 35. Iglesias, 36. Bancos, caja agraria, caja de ahorros, 37. Servicios médicos, 38. Almacenes 
A veces se pueden encontrar pequeñas fondas en doride los peones toman sus alimentos. Casi siempre en estas localidades existen también cantinas. En cambio es notable la falta de establecimientos culturales. Sólo en el caso de El Chote nos han indicado que existe una sala de baile donde se celebran las fiestas religiosas. En algunas de estas localidades (por ejemplo en Chinampa de Gorostiza, Colomán y Raya Obscura) se encuentran pequeños talleres artesanales (de carpinteria, de producción de canastas, de preparación de hojas de maíz para fines comerciales etc.)

Cuadro 1

Localida'des según número de habitantes

\begin{tabular}{l|c}
\hline Número de habitantes & $\begin{array}{c}\text { Número } \\
\text { de localidades }\end{array}$ \\
\hline Menos de 1000 personas & 11 \\
$1000-2000$ & 6 \\
$2000-5000$ & 3 \\
$5000-10000$ & 6 \\
$10000-15000$ & 2 \\
Más de 15000 & 5 \\
\hline Total & 33 \\
\hline
\end{tabular}

Ad. 3. Dentro del tercer grupo se encuentran localidades que cuentan con servicios básicos más numerosos y con varios tipos de talleres productivos (por ejemplo: panaderías, talleres mecánicos, barberías, carnicerias, triendas de ropa etc.). Aguí se pueden enumerar los siguientes centros poblacionales: Chalahuiyapa, Zacamixtle, Tampache, Juan Felipe, Coacuilco, El Humo, Tantóbal, Ixcatlán y Pujal. El número de habitantes de dichos pueblos oscila entre 1000 y 2000 personas.

De acuerdo con las leyes mexicanas que dividen a la población entre rural y urbana (el límite entre el centro urbano y el rural es de 2500 habitantes) estas localidades se clasifica todavía como rurales, excepto Coacuilco, que tiene 7000 habitantes. Pero resulta, basándonis en la información resultante de las encuestas, que el número de servicios y IV. Producción

IV. Producción

39. Establecimientos industriales

40. Talleres artesanales

V. Infraestructura técnica

41. Electrificación, 42. Acueducto, 43. Alcantarillado 
de otras actividades no agrícolas en Coacuilco está muy limitado y se distingue mucho del número de las mismas actividades que aparecen en los centros urbanos mencionados en el punto 4, por ello se le ha dejado en el tercer grupo.

Ad. 4. Dentro de este grupo se encuentran: El Higo, Piatón Sánchez, Tamuín, Cerro Azul, Naranjos, Tamiahua, Tempoal, Fuejutla, Tepetzintla, Rascón, Tehuetlán y Chalma. Los tres últimos centros urbanos se encuentran entre los grupos tercero y cuarto. Lo que decidió sobre su incorporación al grupo cuarto fue el número de población 6000,3000 y 6500 habitantes respectivamente. De acuerdo con datos oficiales estas localidades ya son centros urbanos. El número de pablación de las otras ciudades integradas a este grupo oscila entre 7000 (en el caso de Tamiahua) y 50000 (el caso de Cerro Azul).

En base del análisis de la estructura de servicios y de las actividades productivas podemos indicar cierta similitud entre dichos centros. Es distinto (a veces significamente) el grado de su densidad. Es posible afirmar, que en las localidades estudiadas el nivel de densidad de los servicios depende del número de la población de tal manera que cuando crece el número de habitantes también crece el número de servicios dentro de la entidad especial. No podemos observar ninguna corelación positiva entre el número de las actividades no productivas y la distancia entre cualquiera de las localidades y su centro regional más cercano. Es decir la presencia de un centro regional, por ejemplo de Cerro Azul o de Huejutla no influye a la presencia o no de algunos tipos de actividaides en los centros poblacionales que los rodean.

Lo que diferencia este grupo de las ciudades es la existencia o la falta de empresas industriales. En algunas de ellas, ahí donde se han creado grandes empresas industriales (Cerro Azul, Tamuín, El Higo, Tempoal) ha cambiado significamente la estructura del empleo, beneficiando por supuesto el sector industrial. El desarrollo de la industria ha provocado aquí también el desarrollo de otros sectores de la economia, sobre todo del transporte, del comercio y de la construcción lo que ha estimulado el aumento del número de empleos en dichos sectores. Este fenómeno ha sido visible sobre todo en Cerro Azul - viejo centro petrolero y en Tamuín donde se han creado cementos de ANAHUAC y La NESTLE.

De manera diferente a las anteriores se distingue en este grupo Huejutla. En esta ciudad y en sus alrededores está desarrollada la artesanía popular. Es el único centro artesanal en toda la región estudiada. De lo que se puede concluir que este tipo de actividad no puede satisfacer las necesidades de la familia, razón por la cual, la artesanía ha sobrevivido solamente en la zona habitada por los indígenas. 
Analizando los datos sobre el empleo en todas las localidaides, hay que decir, que la cantidad de personas que viven de las actividades no agricolas es muy reducida (con excepción de Cerro Azul, Tamuín y Tamiahua). Las empresas ya existentes de varios tipos, tanto productivas como de servicios, no pueden absorber todo el excedente ide la mano de obra existente en la región (con ciertas diferencias en el caso de Huejutla que desde hace tres años está aumentando intensivamente el número de empleos en la construcción). También ahí en donde la saturación de los establecimientos productivos y de servicios no agrícolas es más grande que en otras localidades la situación en este aspecto no es mejor.

Todo esto ha causado que el área ide esitudio se caracterice por indices muy altos de emigración de la población, principalmente de la zona rural. Generalmente éstas son migraciones estacionales. Cuandc empiezan las labores agrícolas desde junio hasta diciembre en la zona de Las Huastecas la gente permanece buscando el trabajo en el lugar donde vive. Desde enero se empiezan movimientos en busca de empleo. El flujo principal de migrantes se dirige hacia la costa atlántica. Allá trabajan en la agricultura (en la cosecha de tomates, cítricos) o en el puerto de Tampico, que atrae el $\mathbf{5 0 \%}$ de todos migrantes del área de estudio. $\mathbf{M u -}$ cha gente va también a Reynosa, Veracruz y al Distrito Federal; hasta hace poco el principal centro inmigratorio ha sido Poza Rica - gran centro petrolero, pero desdie hace algunos años el número de personas que llegan anualmente a ese lugar ha disminuido. Lo que no significa tampoco, que todavía no absorba a los migrantes, al contrario siempre hay personas que se quedan en esta ciudad.

Las migraciones pues, al lado de los elementos económicos favorables al desarrollo regional (la creación de nuevas empresas industriales, nuevos ejidos, desarrollo del comercio etc.) no han resuelto el problema de fuerza del trabajo sobrante en la región estudiada. 
